\title{
Fetal somatic growth trajectory differs by type of congenital heart disease
}

\author{
Kriti Puri ${ }^{1}$, Carri R. Warshak ${ }^{2}$, Mounira A. Habli ${ }^{3}$, Amy Yuan ${ }^{3}$, Rashmi D. Sahay ${ }^{4}$, Eileen C. King ${ }^{4}$, Allison Divanovic ${ }^{1}$ and
} James F. Cnota ${ }^{1}$

BACKGROUND: The growth trajectories of common measurements, including estimated fetal weight (EFW), head circumference $(H C)$, and abdominal circumference $(A C)$, in fetuses with congenital heart disease (CHD) have not been described for different cardiac lesions. We hypothesized that (i) fetuses with CHD have differential growth in utero, and (ii) different categories of $\mathrm{CHD}$ demonstrate different in utero growth curves.

METHODS: We performed a retrospective observational cohort study of pregnancies with known fetal CHD seen from January 2000 to June 2013. For analysis, the infants were divided into single ventricle (SV), biventricular conotruncal, d-transposition of great arteries (d-TGA), biventricular septal defects (SD; including atrial, ventricular, and atrioventricular SD), and all others (Other).

RESULTS: A total of 194 newborns met inclusion criteria. There was significant differential growth of EFW in all CHD types, except d-TGA, starting with low z-scores before 25 weeks gestation, improving toward normal around 30-32 weeks gestation, and then again differential growth with advancing gestation. SV and SD groups had significant differential growth of $\mathrm{HC}$ starting early in gestation and linearly progressing negative z-scores with advancing gestation.

CONCLUSION: We observed differences in the fetal growth curves throughout gestation for the major categories of CHD, including significant differential growth in even "simple" CHD, such as SD.

C ongenital heart disease (CHD) has been associated with poor fetal growth $(1,2)$. Distinct patterns of newborn anthropometry have been described in specific CHD lesions, likely secondary to alterations in circulatory physiology. For instance, a greater head volume as compared with birth weight in case of coarctation of aorta and a disproportionately smaller head volume as compared with birth weight in case of hypoplastic left heart syndrome (HLHS) (2). Previous data also suggest a decrease in the growth velocity during pregnancy in the fetus with HLHS and other single ventricle
(SV) defects $(3,4)$. However, the fetal trajectory of various somatic growth parameters such as estimated fetal weight (EFW), femur length (FL), head circumference (HC), and abdominal circumference (AC) has not been described for most cardiac lesions. Evidence of early growth restriction has been associated with an increased risk of adverse pregnancy outcomes, including increased perinatal mortality, lower birth weight, or increased congenital malformations (5-7). A better understanding of in utero growth in CHD may influence obstetric decision making and antenatal care. It may also provide an insight into the circulatory pathophysiology of various lesions, beyond the single measurement of a birth weight alone.

The primary aim of this study was to describe somatic growth trajectories of fetuses with CHD. We hypothesized that (i) fetuses with CHD have differential growth in utero, as seen by a difference in the $z$-scores of the anthropometry parameters at serial measurements, and (ii) different categories of CHD based on physiology will demonstrate different growth curves in utero.

\section{METHODS}

\section{Study population}

This was a retrospective observational cohort study that included infants with prenatally diagnosed CHD, whose mothers' received obstetric care at the University of Cincinnati Medical Center and the Good Samaritan Hospital in Cincinnati, and fetal prenatal checks at Cincinnati Children's Hospital Medical Center between January 2000 and June 2013. Exclusion criteria included-(i) antenatal or postnatal diagnosis of known genetic anomaly or syndrome that are known to be associated with growth restriction and could be a confounder (trisomy 13, trisomy 18, trisomy 21, Turner syndrome, deletion 22q11.2), (ii) hydrops of any cause (causing potential error in correct assessment of somatic measurements), (iii) hydrocephalus of any cause (causing potential error in correct assessment of HC), and (iv) multiple gestations (to avoid confounding as duration of gestation and weight is known to be lower in these). Subjects were also excluded if a minimum of two data points of growth measurements were not available. Ethical approval was obtained from the Institute Review Board of all three involved institutes.

\section{Data collection}

Demographic characteristics of the mothers, as well as risk factors such as diabetes before and during pregnancy, preeclampsia, and

\footnotetext{
${ }^{1}$ The Heart Institute, Cincinnati Children's Hospital Medical Center, Cincinnati, Ohio; ${ }^{2}$ Maternal-Fetal Medicine at the Cincinnati Fetal Center, Cincinnati Children's Hospital Medical Center, Cincinnati, Ohio; ${ }^{3}$ Department of Obstetrics and Gynecology, Good Samaritan Hospital, Cincinnati, Ohio; ${ }^{4}$ Department of Biostatistics and Epidemiology, Cincinnati Children's Hospital Medical Center, Cincinnati, Ohio. Correspondence: James F. Cnota (james.cnota@cchmc.org) 


\section{Articles | Puri et al.}

smoking status were collected. EFW was calculated using the Hadlock method (1984), while fetal measurements, including FL, $\mathrm{HC}$, and AC, were collected from serial obstetric ultrasounds throughout gestation (8). Birth measurements for weight, length, HC, and presence of single umbilical artery were recorded from delivery reports. Cases of intrauterine fetal demise were included as long as at least two measurements of growth parameters were available. Gestational age was obtained from the obstetric records, as determined by the best clinical estimate that relied upon the last menstrual period when an ultrasound prior to 22 weeks was within

Table 1. Demographic features and pregnancy characteristics of mother-fetus dyads with congenital heart disease

\begin{tabular}{|c|c|c|c|c|c|c|}
\hline Variables & $\begin{array}{l}\text { Single ventricle } \\
(n=64)\end{array}$ & $\begin{array}{c}\text { Conotruncal defects } \\
(n=45)\end{array}$ & d-TGA $(n=9)$ & $\begin{array}{l}\text { Septal defects } \\
\quad(n=29)\end{array}$ & Other $(n=47)$ & $P$-value ${ }^{a}$ \\
\hline Sex, $n(\%)$ & & & & & & 0.02 \\
\hline Female & $18(28.1)$ & $22(50.0)$ & $2(22.2)$ & $17(60.7)$ & $20(43.5)$ & \\
\hline Male & $46(71.9)$ & $22(50.0)$ & $7(77.8)$ & $11(39.3)$ & $26(56.5)$ & \\
\hline Missing, $n$ & 0 & 1 & 0 & 1 & 1 & \\
\hline Hispanic & $2(3.8)$ & $0(0)$ & $0(0)$ & $0(0)$ & $2(4.9)$ & \\
\hline Non-Hispanic & $51(96.2)$ & $38(100)$ & $8(100)$ & $23(100)$ & $39(95.1)$ & \\
\hline Missing, $n$ & 11 & 7 & 1 & 6 & 6 & \\
\hline Missing, $n$ & 8 & 6 & 0 & 7 & 5 & \\
\hline Smoking, n (\%) & & & & & & 0.92 \\
\hline No & $44(68.8)$ & $27(60.0)$ & $7(77.8)$ & $18(62.1)$ & $29(61.7)$ & \\
\hline Yes & $10(15.6)$ & $9(20.0)$ & $0(0)$ & $6(20.7)$ & $8(17.0)$ & \\
\hline Unknown & $10(15.6)$ & $9(20.0)$ & $2(22.2)$ & $5(17.2)$ & $10(21.3)$ & \\
\hline Preeclampsia, $n$ (\%) & & & & & & 0.86 \\
\hline No & $54(90)$ & $39(86.7)$ & $7(100)$ & $24(85.7)$ & $42(91.3)$ & \\
\hline Yes & $3(4.8)$ & $7(15.6)$ & $1(14.3)$ & $0(0)$ & $2(4.4)$ & \\
\hline Missing, $n$ & 2 & 0 & 2 & 2 & 2 & \\
\hline Gestational diabetes, $n$ (\%) & & & & & & 0.75 \\
\hline No & $55(93.2)$ & $41(91.1)$ & $6(85.7)$ & $27(96.4)$ & $41(91.1)$ & \\
\hline Yes & $4(6.8)$ & $4(8.9)$ & $1(14.3)$ & $1(3.6)$ & $4(8.9)$ & \\
\hline Missing, $n$ & 5 & 0 & 2 & 1 & 2 & \\
\hline SUA, $n(\%)$ & & & & & & 0.71 \\
\hline No & $49(76.6)$ & $36(80.0)$ & $6(66.7)$ & $21(72.4)$ & $34(72.3)$ & \\
\hline Yes & $7(10.9)$ & $3(6.7)$ & $1(11.1)$ & $6(20.7)$ & $7(14.9)$ & \\
\hline Unknown & $8(12.5)$ & $6(13.3)$ & $2(22.2)$ & $2(6.9)$ & $6(12.8)$ & \\
\hline
\end{tabular}

d-TGA, d-transposition of great arteries; SUA, single umbilical artery.

${ }^{a}$ Chi-square/Fisher's test used to examine significant differences among the groups 
10 days of clinical dating or re-dated when $>10$ days difference from clinical dating (9). CHD was confirmed and described using fetal echocardiography and classified based on a tri-level nomenclature previously described, incorporating the Society of Thoracic Surgeons' classification as the most specific level, level 1 , and then broader levels 2 and 3 that incorporate the embryological origins (10). For purpose of analysis, CHDs were collapsed into fewer categories, namely, SV, biventricular conotruncal, d-transposition of great arteries (d-TGA), biventricular septal defects (SD, including atrial and ventricular septal defects and atrioventricular SD), and all other CHD (Others). This categorization was performed for purposes of statistical analysis and was influenced by the physiology of blood flow and the clinical manifestation and management principles in these groups of lesions.

To standardize the fetal biometry and birth anthropometry measurements for given gestational age, $z$-scores were calculated. $z$-Score for EFW and HC were calculated using the least mean square parameters for gender-specific growth curves presented by Olsen et al. (11), therefore gestational age was restricted to $23-41$ weeks. The $z$-weight and $z$-HC at birth were plotted as the final data points when creating these curves. To compute $z$-scores for $\mathrm{FL}$ and $\mathrm{AC}$, international standards for fetal growth from INTERGROWTH-21st were used and therefore early weeks of gestation were examined (12).

\section{Statistical analysis}

Descriptive statistics were presented as frequencies and percentages for categorical and as median and interquartile range for continuous variables. If the number of missing observations for maternal-fetal risk factors was $>10 \%$ of the total sample number, then an unknown category was created for analysis. Significant differences in the characteristics among CHD groups were examined by Chi-square/ Fisher's exact test for categorical variables and Kruskal-Wallis test for continuous variables. To examine growth trajectories of weight, $\mathrm{FL}, \mathrm{HC}$, and $\mathrm{AC}$ over time for each of the CHD type, multilevel mixed-effect modeling was carried out. Linear and quadratic models were developed, and growth trajectories for each outcome was determined by testing for significance of the linear and quadratic terms in the models. As $z$-scores were calculated to standardize fetal biometry and birth anthropometry measurements for given gestational age using a normal reference population, trajectories above the $z$-score of zero represents growth higher than normal and those below zero represents growth lower than normal reference population. Sex, pregestational diabetes, and maternal smoking were found to be significant covariates in the univariate analyses and were therefore adjusted for in the multivariable models. Results were presented as estimates and SE, with $P$-values indicating whether the coefficient for the linear or quadratic term for given CHD type is significantly different than zero. All analyses were conducted using SAS version 9.4 (SAS Institute, Cary, NC). Two-sided testing was carried out and $P$-value $\leqslant 0.05$ was considered statistically significant.

\section{RESULTS}

A total of 194 newborns met inclusion criteria. The details of the diagnoses among each of the groups are shown in Supplementary Appendix S1.

The patient demographics are described in Table 1. Significant association was observed between sex and CHD types $(P=0.02)$. SV and d-TGA groups had a predominance of males, and SD had a predominance of females. None of the maternal factors studied demonstrated significant association with types of CHD. The unadjusted anthropometry measurements at birth, by CHD types, are presented in Table 2. $z$-Scores for weight were significantly different among $\mathrm{CHD}$ types. None of the other measures were significantly different.

\section{Trajectories}

The unadjusted patterns of growth curves (intrauterine to birth) for weight, FL, HC, and AC, expressed as plots of the $z$ scores of the measurement over time of gestation, are represented in Figures 1,2,3,4, respectively. Each figure includes the overall plot comparing different categories of $\mathrm{CHD}$, as well as individual plots showing all the patients in each category of CHD. The coefficients for growth parameters by $\mathrm{CHD}$ type after adjusting for maternal-fetal factors are presented in Table 3.

Weight. The growth curves for weight showed significant curvilinear negative relationship in all CHD types, except d-TGA (Figure 1). Although the curve for d-TGA appears to have differential growth, the results, however, were not significant either in the univariate or the multivariable model. EFW was lower than the reference population before 25 weeks

Table 2. Birth anthropometry of study infants with congenital heart disease

\begin{tabular}{|c|c|c|c|c|c|c|c|c|c|c|c|}
\hline \multirow[t]{2}{*}{ Anthropometry } & \multicolumn{2}{|c|}{ Single ventricle } & \multicolumn{2}{|r|}{$\begin{array}{c}\text { Conotruncal } \\
\text { defects }\end{array}$} & \multicolumn{2}{|r|}{ d-TGA } & \multicolumn{2}{|r|}{ Septal defects } & \multicolumn{2}{|r|}{ Other } & \multirow[t]{2}{*}{$P_{\text {-value }}{ }^{a}$} \\
\hline & $n$ & & $n$ & & $n$ & & $n$ & & $n$ & & \\
\hline GA (weeks) & 64 & 39 (37-39) & 45 & $38(36-39)$ & 9 & $39(36-39)$ & 29 & $37(36-39)$ & 47 & $38(36-39)$ & 0.61 \\
\hline Weight (kg) & 61 & $3(2.7-3.3)$ & 44 & $2.7(2.2-3.3)$ & 9 & $3.2(3.2-3.4)$ & 27 & $2.9(2.4-3.2)$ & 44 & $2.9(2.5-3.3)$ & 0.15 \\
\hline z-Weight & 61 & $-0.7(-1.1,0.2)$ & 44 & $-0.9(-1.9,0)$ & 9 & $0(0,0.1)$ & 27 & $-0.3(-0.9,0.3)$ & 44 & $-0.4(-0.8,0.5)$ & 0.02 \\
\hline $\mathrm{HC}(\mathrm{cm})$ & 46 & $34(32-34.5)$ & 32 & $33(31.5-35)$ & 6 & $33(31.5-33.5)$ & 16 & $32.5(31.5-34)$ & 33 & $34(32-35)$ & 0.34 \\
\hline$z$-Head circumference & 46 & $-0.2(-1.3,0.2)$ & 32 & $-0.4(-1.3,0.4)$ & 6 & $-0.4(-1.4,-0.1)$ & 16 & $0(-0.9,0.7)$ & 33 & $-0.1(-1.1,0.6)$ & 0.70 \\
\hline Length $(\mathrm{cm})$ & 51 & $48.5(46-51)$ & 35 & $47(44-50)$ & 6 & $48.5(47-50)$ & 18 & $48.2(44.5-50.5)$ & 36 & $47.9(45.3-50.8)$ & 0.53 \\
\hline Apgar-1 min & 59 & $8(6-8)$ & 43 & $8(6-8)$ & 9 & $8(7-8)$ & 25 & $8(6-8)$ & 45 & $8(8-9)$ & 0.30 \\
\hline Apgar $-5 \mathrm{~min}$ & 58 & $9(8-9)$ & 43 & $9(8-9)$ & 9 & $8(8-8)$ & 25 & $9(8-9)$ & 45 & $9(8-9)$ & 0.37 \\
\hline
\end{tabular}

d-TGA, d-transposition of great arteries; GA, gestational age; HC, head circumference. 

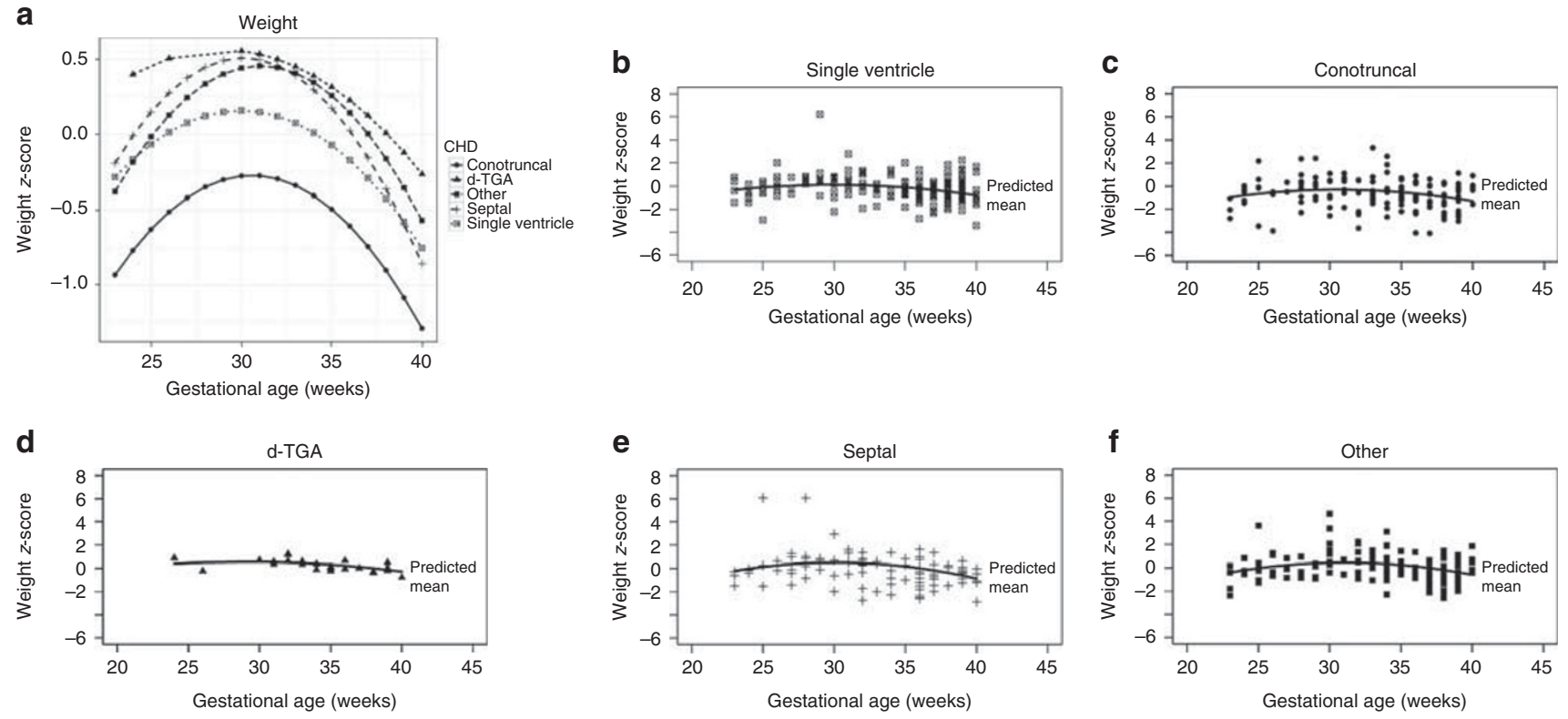

Figure 1. Growth trajectories for weight by category of congenital heart disease. Individual figures show plots of weight in each congenital heart disease category, with each marker representing one patient observation. (a) Growth trajectories of weight by category of congenital heart disease, complete cohort; (b) growth trajectory of weight in the "single ventricle" group; (c) growth trajectory of weight in the "conotruncal defects" group; (d) growth trajectory of weight in the d-transposition of great arteries ("d-TGA") group; (e) growth trajectory of weight in the "septal defects" group; (f) growth trajectory of weight in the "Others" group.
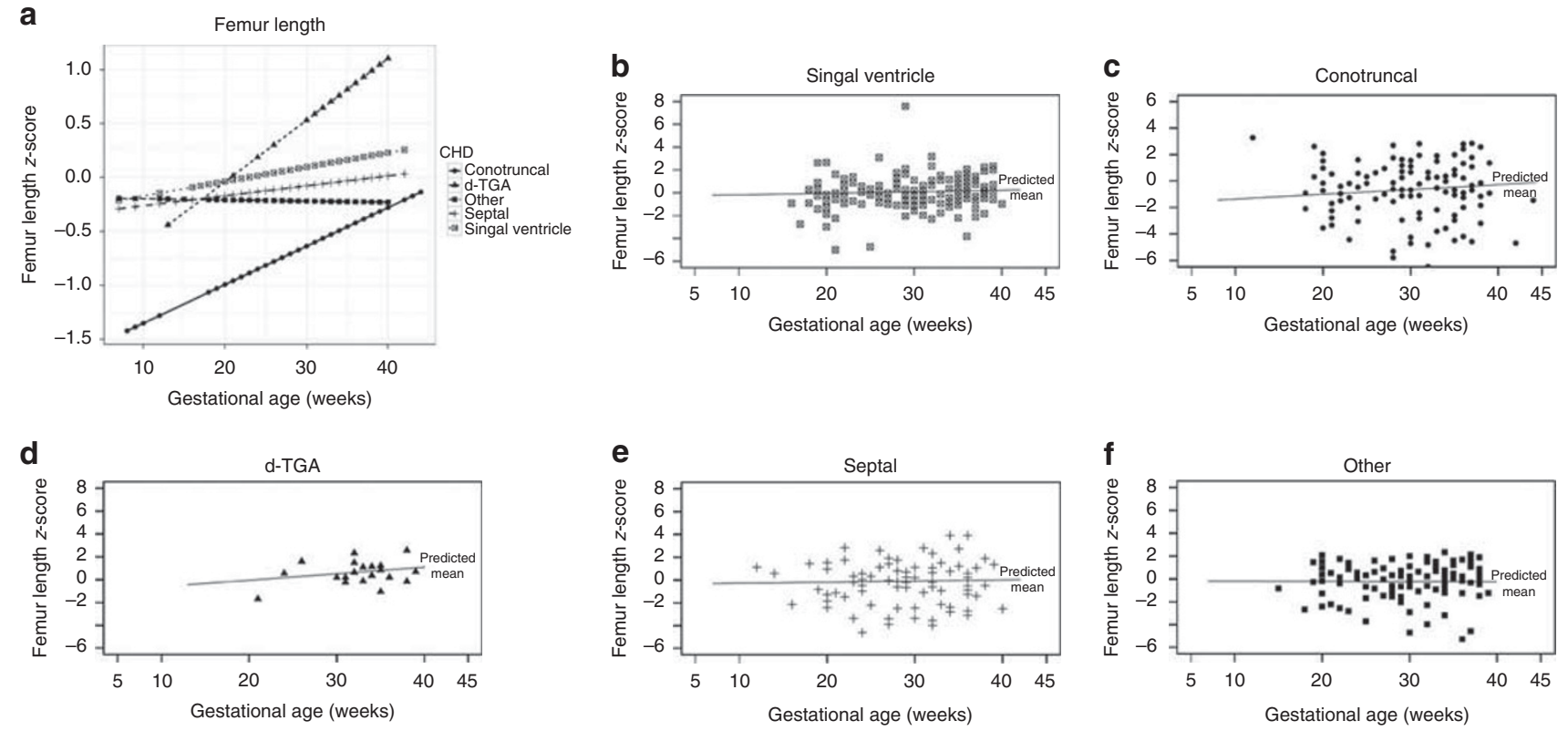

Figure 2. Growth trajectories for femur length by category of congenital heart disease. Individual figures show plots of femur length in each congenital heart disease category, with each marker representing one patient observation. (a) Growth trajectories of femur length by category of congenital heart disease, complete cohort; (b) growth trajectory of femur length in the "single ventricle" group; (c) growth trajectory of femur length in the "conotruncal defects" group; (d) growth trajectory of femur length in the d-transposition of great arteries ("d-TGA") group; (e) growth trajectory of femur length in the "septal defects" group; (f) growth trajectory of femur length in the "Others" group.

of gestation, with improving $z$-scores toward a plateau around 28-32 weeks and then again showed progressively negative $z$-score below the reference population, indicating resumption and persistence of differential growth with advancement of gestation into the third trimester. Similar results were present in the adjusted models. The final data value plotted for EFW is the birthweight, and the correlation between the birth weight and the last EFW was $r=0.50, P<0.001$.

Femur length. Linear positive growth was observed for FL in all CHD types; however, none of the coefficients were found to be significant in the adjusted models (Figure 2). 

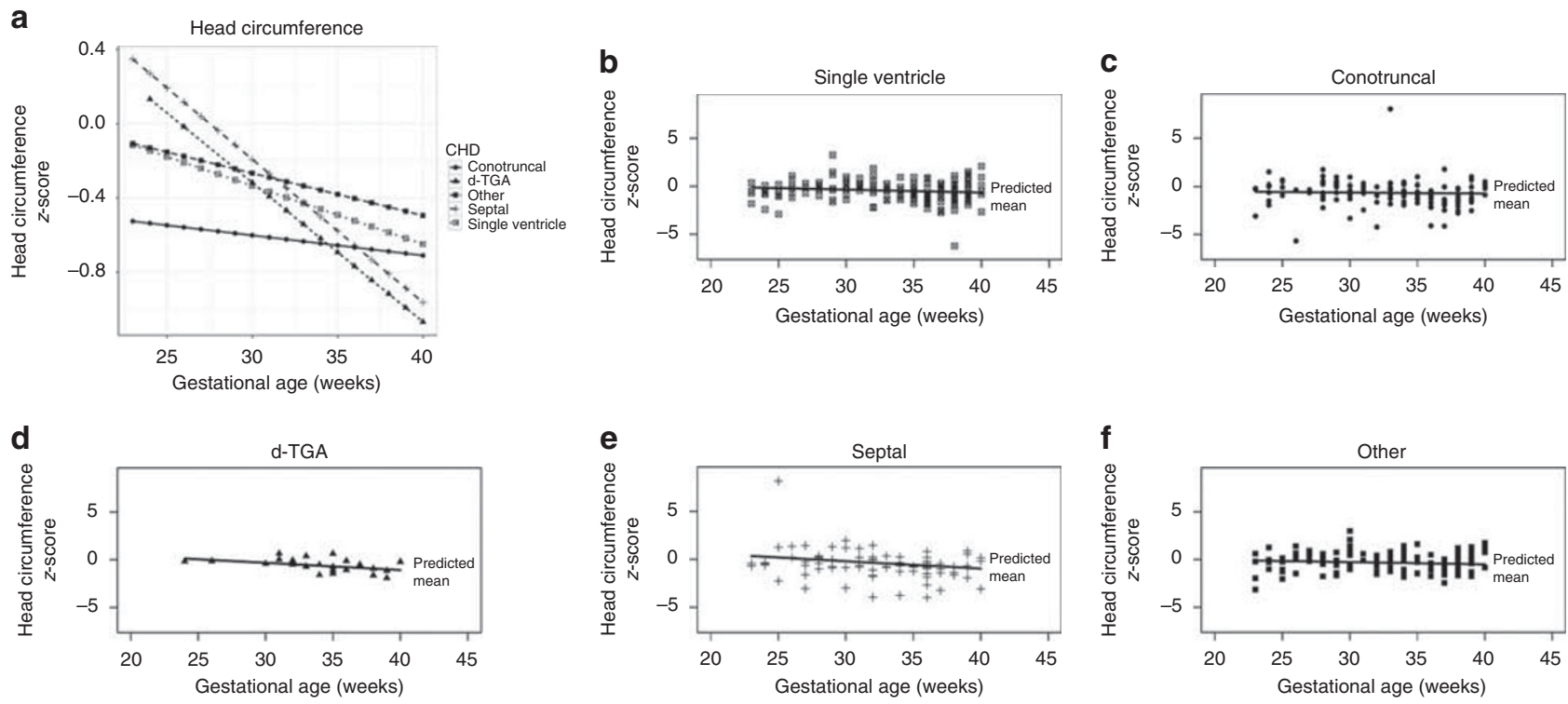

Figure 3. Growth trajectories for head circumference by category of congenital heart disease. Individual figures show plots of head circumference in each congenital heart disease category, with each marker representing one patient observation. (a) Growth trajectories of head circumference by category of congenital heart disease, complete cohort; (b) growth trajectory of head circumference in the "single ventricle" group; (c) growth trajectory of head circumference in the "conotruncal defects" group; (d) growth trajectory of head circumference in the d-transposition of great arteries ("d-TGA") group; (e) growth trajectory of head circumference in the "septal defects" group; (f) growth trajectory of head circumference in the "Others" group.
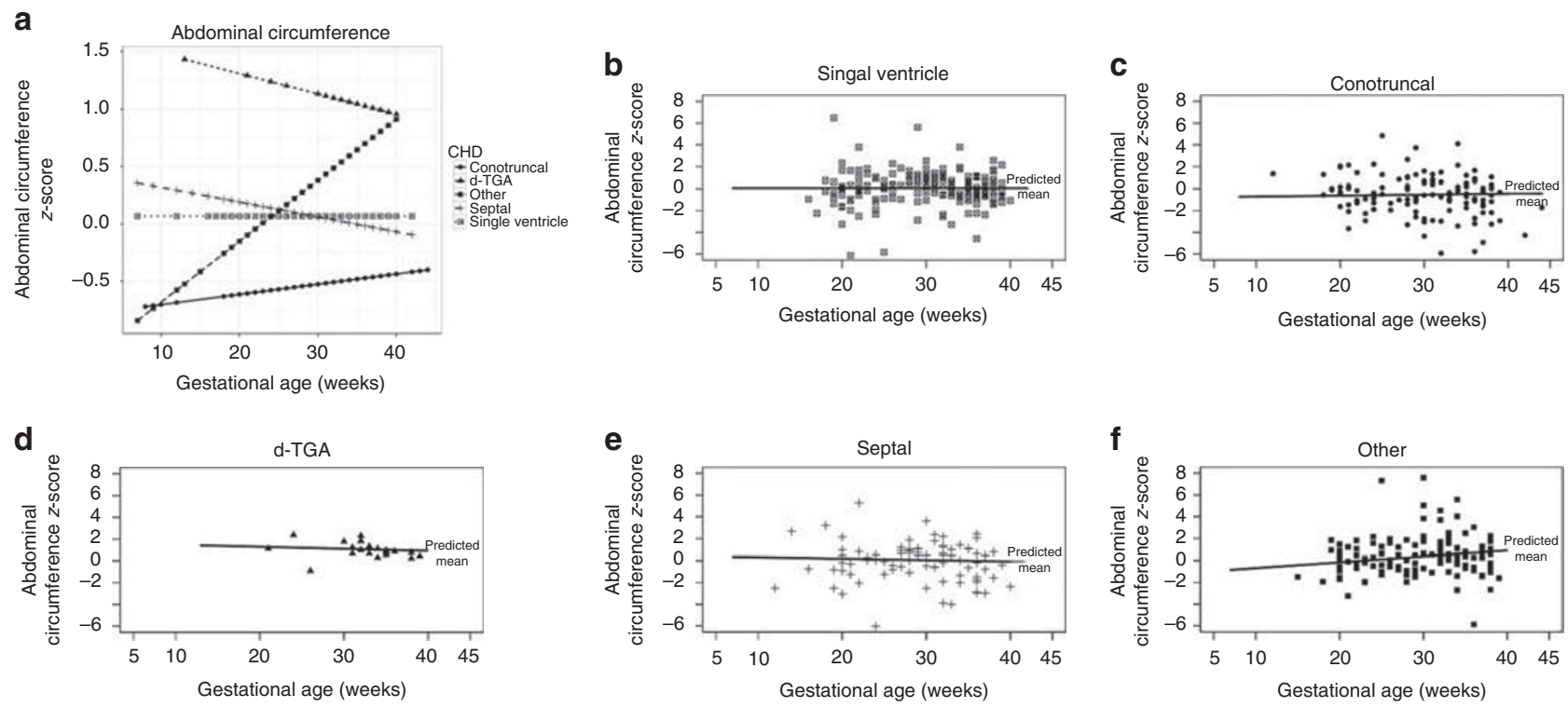

Figure 4. Growth trajectories for abdominal circumference by category of congenital heart disease. Individual figures show plots of abdominal circumference in each congenital heart disease category, with each marker representing one patient observation. (a) Growth trajectories of abdominal circumference by category of congenital heart disease, complete cohort; (b) growth trajectory of abdominal circumference in the "single ventricle" group; (c) growth trajectory of abdominal circumference in the "conotruncal defects" group; (d) growth trajectory of abdominal circumference in the d-transposition of great arteries ("d-TGA") group; (e) growth trajectory of abdominal circumference in the "septal defects" group; (f) growth trajectory of abdominal circumference in the "Others" group.

Head circumference. For all CHD types, linear negative growth curves for HC were found. In the adjusted models, coefficients remained negative and were found to be significant for SV and SD (Figure 3).
Abdominal circumference. The growth curves for AC showed a linear relationship with respect to gestational age and in the adjusted models results remained significant for the "Others" CHD group (Figure 4). 
Table 3. Coefficients for model terms ${ }^{a}$ for each growth parameter by congenital heart disease types

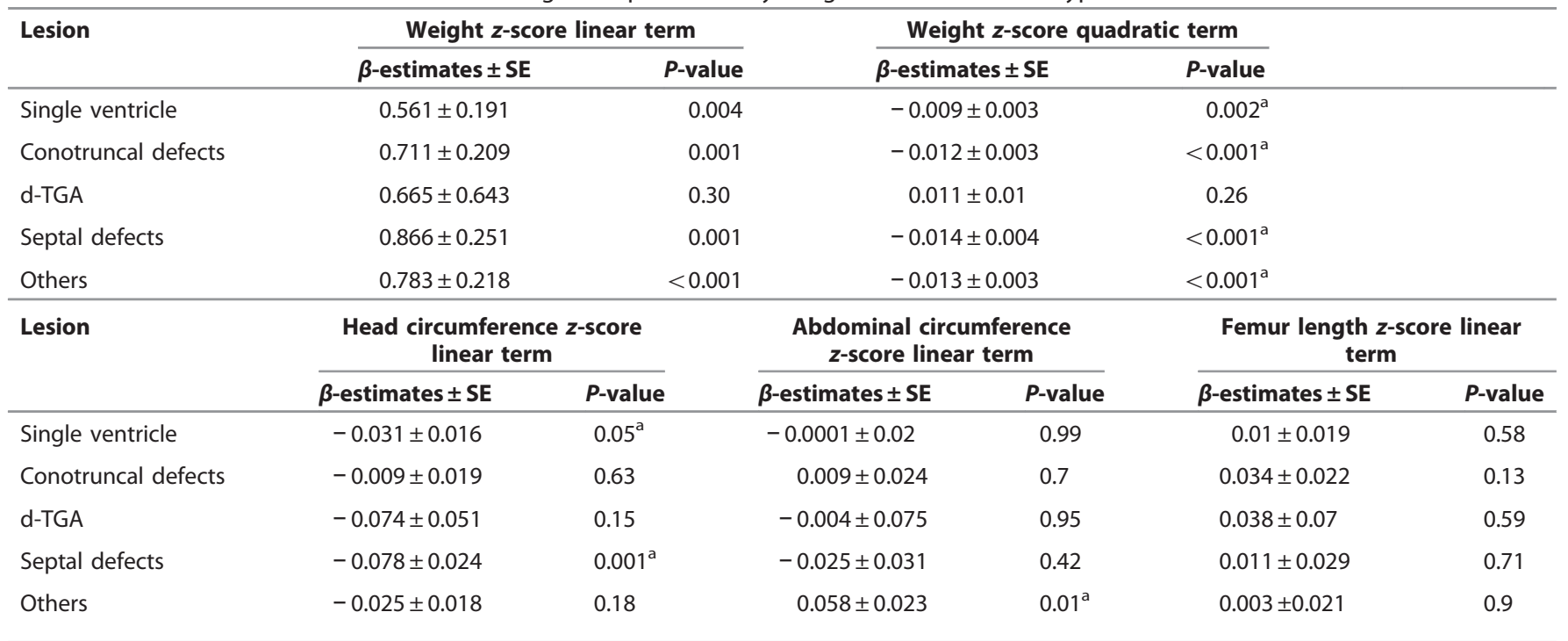

d-TGA, d-transposition of great arteries. All models were adjusted for sex, pregestational diabetes, and maternal smoking.

${ }^{a}$ Quadratic models were estimated for weight. Linear models were estimated for head circumference, abdominal circumference, and femur length.

\section{DISCUSSION}

We report a distinct pattern of differential somatic growth starting in the early trimester, slowing down in the middle, and then resuming and progressing in the third trimester again, in fetuses with most types of $\mathrm{CHD}$, including varieties that are classically considered simple lesions. We also found the $\mathrm{HC}$ growth to be significantly impaired from early gestation at $<24$ weeks and continue to be lower throughout gestation in the SD and SV groups.

The role of biometry of the fetus and newborn in the field of CHD is evolving rapidly. Lower birth weight has been found to be associated with greater risk of poor outcomes such as death or complications following corrective cardiac surgery $(5,13)$. Smaller infants with CHD are also at higher risk of adverse neurodevelopmental outcomes in the long term $(4,14)$. Several studies have found a significant association between the presence of $\mathrm{CHD}$ and lower birth weight and intrauterine growth restriction $(1,2)$. However, neonatal measurements are only a single point in time. In this study, $94 \%$ of subjects had at least three data points, two during fetal period and one at delivery. Understanding the in utero growth trajectory may provide additional pathophysiological insights about how the prenatal course shapes subsequent outcomes.

Along with birth weight, the prognostic role of $\mathrm{HC}$ throughout gestation and at birth is also a topic of significant research. A global decrease in cerebral volume in newborns with CHD has been reported in postnatal studies (15). Hahn et al. (4) found the HC $z$-score in fetuses with SV CHD significantly $<0$ throughout gestation and noted a significant decrease in growth rate in the later gestation period between 24-29 and 34 weeks. However, the intrauterine pattern of somatic growth in fetuses with anatomically and physiologically different categories of CHD has not been previously contrasted and reported. We found a significant downtrend throughout gestation in the HC measures in the SV and SD groups. The SV group is an established high-risk group and the focus of significant ongoing neurodevelopmental outcomes' research. Our findings indicate that the SD group, with diagnoses including atrial and ventricular SD that are traditionally considered less complex, are also at significant risk for differential growth and need to be included into the realm of focused long-term neurodevelopmental monitoring.

We found significantly differential growth of EFW in all the groups of CHD except the d-TGA group. This is consistent with clinical observations that infants with d-TGA have preserved birth weight or are large for gestational age, often in the setting of maternal diabetes $(1,16)$. In our study, maternal diabetes did not have a significant association with type of CHD, possibly due to smaller numbers. Hahn et al. (4) found that, in SV patients, the EFW $z$-score remained in the normal range until 33 weeks gestation when it dropped to $<0$. We observed that the EFW $z$-scores have an early onset of differential growth, which improves with $z$-score approaching toward the normal until about 30 weeks gestation, before resuming a downward slope. This coincides with the time period at which maternal-fetal monitoring is more rigorous, and more frequent checks and scans are performed on the mother-baby dyad with known CHD. This also corresponds with the time of rapid fetal growth and cardiac output demands in the normal fetus. In the setting of CHD, it appears that this acceleration of growth does not occur in the normal manner. This may also present a window of opportunity for nutritional and medical intervention to help improve the growth and maturational outcomes.

Williams et al. (17) compared fetal biometry of babies with HLHS, TGA, or tetralogy of Fallot with controls without CHD. They found that early in gestation (18-26 weeks), 
fetuses with CHD were significantly smaller in terms of EFW, FL, HC and AC. At 26-33 weeks, only the AC was significantly lower in CHD as compared with controls. At 34-40 weeks, the EFW, HC and AC were persistently lower in the fetuses with CHD. They also found that the tetralogy of Fallot infants had significantly lower HC as compared with other groups after 27 weeks of gestation and a significantly lower AC and EFW at the measures between 27 and 33 weeks of gestation. We noted the significant differential growth of EFW in our conotruncal group but not in other growth measures of HC, AC, or FL. However, our CHD groups included all variants of SV patients and not just HLHS. The observed differences in CHD types provide further support that researchers must be cautious when combining CHD subtypes in studies of pregnancy outcomes.

Hahn et al. (4) did not note any effect of the heart disease on the FL or the AC throughout gestation in their study on SV patients (4). In our study also, none of the trajectories for FL were significantly different from normal. AC trajectories for SV, conotruncal, d-TGA, and SD groups are also similar to the normal population. This suggests that the differences across subgroups, whether related to physiology, genetic etiology, or maternal factors, have less impact on linear growth, abdominal organs, and adiposity. It is unclear why the AC $z$-score was significantly improving throughout gestation in the "Others" group, though this was a heterogeneous group with multiple types of CHD.

We did not find any association of preeclampsia or maternal smoking with growth outcomes in fetuses with CHD. Previously, preeclampsia has been associated with intrauterine growth restriction, in the setting of dysfunctional placental vascular development and function (18). Further, pregnancies with preeclampsia have also been reported to be associated with severe CHD (19). Jones et al. (20) found that the placentae in infants with HLHS have significantly lower placental weight and lower terminal villous and vascular density. Cigarette smoking also has a known impact on fetal growth with selectively impaired AC and peripheral muscle mass (21).

Our study was retrospective in nature and based on analysis of clinically reported ultrasonography findings carried out as a routine procedure and not on a predefined schedule, thus introducing possibility of measurement bias. Owing to the retrospective nature of data collection, the information available was limited by the documentation in the patient's chart. Although we collected information about hypertensive diseases of pregnancy, we did not collect information regarding structural or functional heart disease in the mother, which may confound fetal growth. As the study was carried out at a tertiary center, there may be referral bias. The final measurements of birth anthropometry are technically different from the in utero measurements, however, were analyzed together in this study. The d-TGA group showed preserved growth of EFW; however, the number of patients was small and only two patients had anthropometric data available before 30 weeks of gestation. Further, we created an "Others" group that was heterogenous as it included small numbers of patients who had diagnoses that could not be placed in any of the other groups. A larger sample size would allow creation of different groups with adequate numbers instead of having to combine these groups. When excluding patients with known genetic anomalies, we included testing carried out in the prenatal period and in the neonatal admission, but not any testing carried out later in life. Further studies tracking individual organ growth trajectories may also reveal differential growth as an important contributor to overall low EFW and growth impairment.

We identified differing growth trajectories among categories of CHD. It is unclear whether this is related to hemodynamic, genetic, or other differences in patient groups. The information may guide clinicians monitoring early fetal growth in the setting of known CHD and improve risk stratification. Furthermore, the observations may direct future research to investigate why different defects lead to these patterns and inform clinical interventions in the future.

\section{SUPPLEMENTARY MATERIAL}

Supplementary material is linked to the online version of the paper at http://www.nature.com/pr

\section{ACKNOWLEDGMENTS}

We are grateful to the staff in the ultrasound and echocardiography laboratories at the involved institutes, as well as to the patients for whom we have the privilege to provide care.

Disclosure: The authors declare no conflict of interest.

\section{REFERENCES}

1. Rosenthal GL, Wilson PD, Permutt T, Boughman JA, Ferencz C. Birth weight and cardiovascular malformations: a population-based study. The Baltimore-Washington Infant Study. Am J Epidemiol 1991;133: 1273-81.

2. Rosenthal GL. Patterns of prenatal growth among infants with cardiovascular malformations: possible fetal hemodynamic effects. Am J Epidemiol 1996;143:505-13.

3. Cnota JF, Hangge PT, Wang Y, et al. Somatic growth trajectory in the fetus with hypoplastic left heart syndrome. Pediatr Res 2013;74:284-9.

4. Hahn E, Szwast A, Cnota J, et al. The association of fetal growth, cerebral blood flow, and neurodevelopmental outcome in single ventricle fetuses. Ultrasound Obstet Gynecol 2015;47:460-5.

5. Ades AM, Dominguez TE, Nicolson SC, et al. Morbidity and mortality after surgery for congenital cardiac disease in the infant born with low weight. Cardiol Young 2010;20:8-17.

6. Nikkilä A, Källén B, Marsál K. Fetal growth and congenital malformations. Ultrasound Obstet Gynecol 2007;29:289-95.

7. Källén K. Increased risk of perinatal/neonatal death in infants who were smaller than expected at ultrasound fetometry in early pregnancy. Ultrasound Obstet Gynecol 2004;24:30-4.

8. Hadlock FP, Harrist RB, Sharman RS, Deter RL, Park SK. Estimation of fetal weight with the use of head, body, and femur measurements-a prospective study. Am J Obstet Gynecol 1985;151:333-7.

9. Committee on Obstetric Practice, the American Institute of Ultrasound in Medicine, and the Society for Maternal-Fetal Medicine. Committee Opinion No 700: methods for estimating the due date. Obstet Gynecol 2017;129:e150-4.

10. Miller A, Riehle-Colarusso T, Alverson CJ, Frías JL, Correa A. Congenital heart defects and major structural noncardiac anomalies, Atlanta, Georgia, 1968 to 2005. J Pediatr 2011;159(1):70-78.e2. 


\section{Articles | Puri et al.}

11. Olsen IE, Groveman SA, Lawson ML, Clark RH, Zemel BS. New intrauterine growth curves based on United States data. Pediatrics 2010;125:e214-24.

12. International Fetal and Newborn Growth Consortium for the 21st century Article: International Fetal Growth Standards - latest charts available - INTERGROWTH-21st https://intergrowth21.tghn.org/articles/ international-fetal-growth-standards-latest-charts-available/ Published 2015. Accessed 22 January 2016.

13. Hickey EJ, Nosikova Y, Zhang H, et al. Very low-birth-weight infants with congenital cardiac lesions: is there merit in delaying intervention to permit growth and maturation? J Thorac Cardiovasc Surg 2012;143: 126-36.

14. Tabbutt S, Gaynor JW, Newburger JW. Neurodevelopmental outcomes after congenital heart surgery and strategies for improvement. Curr Opin Cardiol 2012;27:82-91.

15. von Rhein M, Buchmann A, Hagmann C, et al. Severe congenital heart defects are associated with global reduction of neonatal brain volumes. J Pediatr 2015;167(6):1259-63.e1.
16. Reller MD, Strickland MJ, Riehle-Colarusso T, Mahle WT, Correa A. Prevalence of congenital heart defects in metropolitan Atlanta, 19982005. J Pediatr 2008;153:807-13.

17. Williams IA, Fifer WP, Andrews H. Fetal growth and neurodevelopmental outcome in congenital heart disease. Pediatr Cardiol 2015;36:1135-44.

18. Lin S, Leonard D, Co MAM, et al. Pre-eclampsia has an adverse impact on maternal and fetal health. Transl Res 2015;165:449-63.

19. Brodwall K, Leirgul E, Greve G, et al. Possible common aetiology behind maternal preeclampsia and congenital heart defects in the child: a Cardiovascular Diseases in Norway Project Study. Paediatr Perinat Epidemiol 2016;30:76-85.

20. Jones HN, Olbrych SK, Smith KL, et al. Hypoplastic left heart syndrome is associated with structural and vascular placental abnormalities and leptin dysregulation. Placenta 2015;36:1078-86.

21. Sullivan PM, Dervan LA, Reiger S, Buddhe S, Schwartz SM. Risk of congenital heart defects in the offspring of smoking mothers: a population-based study. J Pediatr 2015;166:978-84. 